\title{
FILLINGS OF UNIT COTANGENT BUNDLES
}

\author{
STEVEN SIVEK AND JEREMY VAN HORN-MORRIS
}

\begin{abstract}
We study the topology of exact and Stein fillings of the canonical contact structure on the unit cotangent bundle of a closed surface $\Sigma_{g}$, where $g$ is at least 2 . In particular, we prove a uniqueness theorem asserting that any Stein filling must be scobordant rel boundary to the disk cotangent bundle of $\Sigma_{g}$. For exact fillings, we show that the rational homology agrees with that of the disk cotangent bundle, and that the integral homology takes on finitely many possible values: for example, if $g-1$ is square-free, then any exact filling has the same integral homology and intersection form as $D T^{*} \Sigma_{g}$.
\end{abstract}

\section{INTRODUCTION}

The unit cotangent bundle $S T^{*} M$ of a Riemannian manifold $M$ is equipped with a canonical contact structure $\xi_{\text {can }}$, given in local coordinates as the kernel of $\alpha=\sum_{i} p_{i} d q_{i}$. The contact manifold $\left(S T^{*} M, \xi_{\text {can }}\right)$ is Stein fillable, with one filling given by the disk cotangent bundle $D T^{*} M$, and it is natural to ask whether other such fillings exist. Our goal in this paper is to study the Stein fillings, and more generally the exact symplectic fillings, of $\left(S T^{*} M, \xi_{\text {can }}\right)$ in the case where $M=\Sigma_{g}$ is a surface of genus $g \geq 2$. We will denote the contact manifold $\left(S T^{*} \Sigma_{g}, \xi_{\text {can }}\right)$ by $\left(Y_{g}, \xi_{g}\right)$.

In the cases $g=0,1$ the fillings of $\left(Y_{g}, \xi_{g}\right)$ are already understood, and we know that in fact any minimal symplectic filling must be diffeomorphic to $D T^{*} \Sigma_{g}$. McDuff [McD90] proved this for $\left(S T^{*} S^{2}=\mathbb{R P}^{3}, \xi_{\text {can }}\right)$, and then Hind Hin00 showed that $D T^{*} S^{2}$ is the unique Stein filling up to Stein homotopy. Similarly, Stipsicz Sti02 proved that a Stein filling of the unit cotangent bundle $S T^{*} T^{2}=T^{3}$ must be homeomorphic to $D T^{*} T^{2} \cong$ $T^{2} \times D^{2}$, and Wendl Wen10] showed that all of its minimal strong symplectic fillings are symplectically deformation equivalent to $D T^{*} T^{2}$.

For $g \geq 2$, however, no such uniqueness results for symplectic fillings are possible. This was observed by Li, Mak, and Yasui [LMY14, Proposition 3.3], who noted that in this case McDuff [McD91] constructed a symplectic 4-manifold which strongly fills its disconnected boundary, one of whose components is $\left(Y_{g}, \xi_{g}\right)$. One can glue a symplectic cap with $b_{2}^{+}$ arbitrarily large to the remaining component to get an arbitrarily large filling of $\left(Y_{g}, \xi_{g}\right)$; or, as pointed out by Wendl Wen14, one can even use this to construct a strong symplectic cobordism from any contact 3-manifold to $\left(Y_{g}, \xi_{g}\right)$.

Despite this, if we require the fillings in question to be exact or Stein then the situation is drastically simpler. Our main results are the following, which appear as Theorem 4.10 and Theorem 3.4 respectively.

Theorem 1.1. If $(W, J)$ is a Stein filling of $\left(Y_{g}, \xi_{g}\right)=\left(S T^{*} \Sigma_{g}, \xi_{\text {can }}\right)$, then $W$ is s-cobordant rel boundary to the disk cotangent bundle $D T^{*} \Sigma_{g}$.

In particular, $W$ is homotopy equivalent rel boundary to $D T^{*} \Sigma_{g}$. 
Theorem 1.2. If $(W, \omega)$ is an exact symplectic filling of $\left(Y_{g}, \xi_{g}\right)=\left(S T^{*} \Sigma_{g}, \xi_{\mathrm{can}}\right)$, then the homology of $W$ is given by

$$
H_{1}(W ; \mathbb{Z}) \cong \mathbb{Z}^{2 g} \oplus \mathbb{Z} / d \mathbb{Z}, \quad H_{2}(W ; \mathbb{Z}) \cong \mathbb{Z}, \quad H_{3}(W ; \mathbb{Z})=0
$$

for some integer $d$ such that $d^{2}$ divides $g-1$, and the intersection form on $H_{2}(W)$ is $\left\langle\frac{2 g-2}{d^{2}}\right\rangle$.

Remark 1.3. The requirement that $d^{2} \mid g-1$ implies that the integral homology and intersection form of an exact filling of $\left(Y_{g}, \xi_{g}\right)$ are uniquely determined (hence isomorphic to those of $D T^{*} \Sigma_{g}$ ) whenever $g-1$ is square-free. This condition is well-known to hold for a subset of the natural numbers with density $\frac{6}{\pi^{2}}$.

Remark 1.4. Li, Mak, and Yasui have independently proved a stronger version of Theorem 1.2. namely that every exact filling of $\left(Y_{g}, \xi_{g}\right)$ has the integral homology and intersection form of $D T^{*} \Sigma_{g}$, by similar arguments. In other words, every exact filling has $d=1$.

One notable feature of Theorems 1.1 and 1.2 is that all of the fillings involved have $b_{2}^{+}(W)$ positive. As far as we are aware, any classification theorems which have been proved to date for symplectic or Stein fillings of fillable contact 3-manifolds $(Y, \xi)$ have the feature that all of the symplectic fillings have $b_{2}^{+}=0$. This is true because the classifications usually follow from one of two starting points: either $(Y, \xi)$ has a symplectic cap containing a symplectic sphere of nonnegative self-intersection, or $(Y, \xi)$ is supported by a planar open book.

In the first of these cases, it follows from McDuff [McD90] that any filling embeds into a blow-up of either $\mathbb{C P}^{2}$ or a ruled surface, and in either case the closed manifold has $b_{2}^{+}=1$, with $H_{2}^{+}$generated by the symplectic sphere inside the cap. In the second case, the classifications use work of Wendl Wen10, who showed that all Stein fillings admit Lefschetz fibrations corresponding to factorizations of the monodromy into positive Dehn twists; but the planarity implies by a result of Etnyre Etn04 (whose proof relies on [McD90]) that the filling is negative definite. These techniques have been applied successfully to many contact structures on lens spaces [McD90, Lis08, PVHM10, Kal13, links of simple singularities OO05, and Seifert fibered spaces [Sta15], among others.

The reason we are able to succeed in the absence of either technique is the use of a CalabiYau cap, as defined and studied by Li, Mak, and Yasui [LMY14]. We find a Lagrangian $\Sigma_{g}$ inside a K3 surface with simply connected complement, and a Weinstein tubular neighborhood of this Lagrangian is symplectomorphic to the disk cotangent bundle of $\Sigma_{g}$, so its complement is a symplectic cap for $\left(Y_{g}, \xi_{g}\right)$. Gluing this cap to any filling produces a closed 4-manifold $X$ of symplectic Kodaira dimension zero, and the classification of the latter [MS97, Bau08, Li06a] tells us that $X$ must be an integral homology K3. In Section 3 we then deduce Theorem 1.2 from careful application of the Mayer-Vietoris sequence, and following this we use properties of Stein fillings in Section 4 to pin down the fundamental group and prove Theorem 1.1 .

Finally, we remark that we would like to strengthen Theorem 1.1 by showing that any Stein filling $W$ of $\left(Y_{g}, \xi_{g}\right)$ is homeomorphic to $D T^{*} \Sigma_{g}$, but for now this may be out of reach using our techniques. This would require a proof of the topological s-cobordism theorem for s-cobordisms between 4-manifolds with fundamental group $\pi_{1}\left(\Sigma_{g}\right)$, which is currently only known when the fundamental group is "good" (see Freedman-Quinn [FQ90]), and it is an open question whether surface groups are good.

Acknowledgments. This work began at the Princeton Low-Dimensional Topology Workshop 2015, and we thank the participants for contributing to a productive environment. 
We thank Matt Day, John Etnyre, Dave Futer and Yo'av Rieck for helpful conversations. We are especially grateful to Ian Agol for pointing out to us that Proposition 4.8 should be true and explaining how it should follow from the RFRS condition. SS was supported by NSF grant DMS-1506157. JVHM was supported in part by Simons Foundation grant No. 279342 .

\section{Calabi-Yau caps}

In this section, we will construct and study a certain type of concave filling which was originally used by Li, Mak, and Yasui [LMY14] to bound the topology of Stein fillings of a given manifold.

Definition 2.1. Let $(Y, \xi)$ be a contact manifold. A Calabi-Yau cap for $(Y, \xi)$ is a symplectic manifold $(W, \omega)$ with concave boundary $(Y, \xi)$ and torsion first Chern class, such that there is a contact form $\alpha$ for $\xi$ and a Liouville vector field $X$ near $Y=\partial W$ satisfying $\alpha=\left.\iota_{X} \omega\right|_{Y}$.

In this section we will show that $\left(Y_{g}, \xi_{g}\right)$ admits a simply connected Calabi-Yau cap by finding an embedded Lagrangian $\Sigma_{g}$ of genus $g$ inside the elliptic surface $E(2)$, which is a K3 surface. The cap $X_{g}$ is then the complement of a Weinstein tubular neighborhood of $\Sigma_{g}$, and it is Calabi-Yau because a K3 surface has trivial canonical class. We remark that Li, Mak, and Yasui construct a Calabi-Yau cap for $\left(Y_{g}, \xi_{g}\right)$ by finding a Lagrangian $\Sigma_{g}$ inside the standard symplectic $T^{4}$, but this larger cap enables us to place much stronger restrictions on the possible fillings.

Theorem 2.2. The elliptic surface $E(2)$ contains a Lagrangian surface $\Sigma_{g}$ of genus $g$ such that the complement $X_{g}$ of a Weinstein tubular neighborhood of $\Sigma_{g}$ is simply connected.

Proof. We express the elliptic fibration $\pi: E(2) \rightarrow S^{2}$ as a fiber sum $E(1) \#_{T^{2}} E(1)$, where if $a$ and $b$ are a pair of curves in the torus $T^{2}$ which intersect exactly once then each fibration $E(1) \rightarrow S^{2}$ has six singular fibers with vanishing cycle $a$ and six with vanishing cycle $b$, corresponding to the relation $(a b)^{6}=1$ in the mapping class group of the torus. We can think of the base of the fibration $\pi$ as a connected sum $S^{2}=S^{2} \# S^{2}$, with one copy of $E(1)$ over each summand.

Let $\gamma \subset S^{2}$ be a simple closed curve separating the two $S^{2}$ summands; then we can arrange for $\gamma$ to have a small collar neighborhood $A=(-\epsilon, \epsilon) \times \gamma \subset S^{2}$, with no critical values of $\pi$, so that the symplectic form on $\pi^{-1}(A) \cong A \times T^{2}$ is the product symplectic form induced by area forms on each factor. In particular, if we pick distinct values $t_{1}, \ldots, t_{g} \in(-\epsilon, \epsilon)$ then the $g$ disjoint tori $T_{i}=\left\{t_{i}\right\} \times \gamma \times a$ are all Lagrangian.

Now let $c \subset S^{2}$ be a matching path [Sei08] between two critical points, one in either $S^{2}$ summand of $S^{2}=S^{2} \# S^{2}$, which each have vanishing cycle $b$. Then $c$ lifts to a Lagrangian sphere $S \subset E(2)$. We can arrange for $c$ to intersect $\gamma$ transversely in a single point, and if each $t_{i}$ is sufficiently close to zero it follows that $S$ intersects each $T_{i}$ transversely in a single point as well, namely the point $a \cap b$ in the fiber above $c \cap\left(\left\{t_{i}\right\} \times \gamma\right)$. We surger $S$ and $T_{i}$ together at each of these points [LS91, Pol91] to produce a Lagrangian $\Sigma_{g}$ of genus $g$. We now take $X_{g}=E(2) \backslash N\left(\Sigma_{g}\right)$, where $N\left(\Sigma_{g}\right)$ is a small Weinstein neighborhood of $\Sigma_{g}$.

It remains to be seen that $X_{g}$ is simply connected. Since $E(2)$ is simply connected, $\pi_{1}\left(X_{g}\right)$ is normally generated by the class of a meridian $\mu$ of the Lagrangian $\Sigma_{g}$. We let $c^{\prime} \subset S^{2}$ be a path in one of the two $S^{2}$ summands with endpoints at a pair of critical values which both have vanishing cycle $a$, such that $c^{\prime}$ intersects $c$ once transversely and is disjoint from each of the $\left\{t_{i}\right\} \times \gamma$. Then there is a sphere $S^{\prime} \subset E(2)$ lying above $c^{\prime}$ (which need not 
be Lagrangian) such that $S^{\prime} \cap S$ is the single point $a \cap b$ in the fiber over $c^{\prime} \cap c$, hence $S^{\prime}$ intersects $\Sigma_{g}$ transversely in precisely this point. We arrange for $S^{\prime}$ to intersect $\overline{N\left(\Sigma_{g}\right)}$ in a single meridional disk $D$ about this point, and then $S^{\prime} \cap X_{g}$ is a disk $\overline{S^{\prime} \backslash D}$ with boundary a meridian of $\Sigma_{g}$, so $[\mu]=0$ and we are done.

Proposition 2.3. The cap $X_{g}$ has Betti numbers $b_{2}^{+}\left(X_{g}\right)=2$ and $b_{2}^{-}\left(X_{g}\right)=19$.

Proof. Recall that $b_{2}^{+}(K 3)=3$ and $b_{2}^{-}(K 3)=19$. If $D_{g}$ is the Weinstein neighborhood of the surface $\Sigma_{g}$, so that $D_{g}$ is symplectomorphic to the disk cotangent bundle of $\Sigma_{g}$, then $H_{2}\left(D_{g}\right)=H_{2}\left(\Sigma_{g}\right)=\mathbb{Z}$, and since $\Sigma_{g}$ has self-intersection $2 g-2>0$ the signature of $D_{g}$ is 1. By Novikov additivity we have $\sigma\left(D_{g}\right)+\sigma\left(X_{g}\right)=\sigma(K 3)=-16$, so $X_{g}$ has signature -17 . It thus suffices to show that $b_{2}^{+}\left(X_{g}\right)=2$.

Let $V_{+} \subset H_{2}(K 3 ; \mathbb{Q})$ be a positive definite 3 -dimensional subspace containing the class $\left[\Sigma_{g}\right]$. We can extend $\left[\Sigma_{g}\right]$ to a rational basis of $V_{+}$whose other two classes are orthogonal to $\left[\Sigma_{g}\right]$, and thus integral multiples of those two classes can be represented by surfaces which are disjoint from $\Sigma_{g}$ and even avoid the neighborhood $D_{g}$. These surfaces span a positive definite subspace of $H_{2}\left(X_{g} ; \mathbb{Q}\right)$, so that $b_{2}^{+}\left(X_{g}\right) \geq 2$. However, if $b_{2}^{+}\left(X_{g}\right) \geq 3$ then adjoining $\left[\Sigma_{g}\right]$ to a basis of a 3 -dimensional positive-definite subspace of $H_{2}\left(X_{g} ; \mathbb{Q}\right)$ would yield $b_{2}^{+}(K 3) \geq 4$, which is absurd.

We can understand the homology of $X_{g}$ more precisely by considering the Mayer-Vietoris sequence associated to the decomposition $K 3=D_{g} \cup_{Y_{g}} X_{g}$.

Proposition 2.4. We have $H_{1}\left(X_{g} ; \mathbb{Z}\right)=H_{3}\left(X_{g} ; \mathbb{Z}\right)=0$ and $H_{2}\left(X_{g} ; \mathbb{Z}\right) \cong \mathbb{Z}^{21} \oplus \mathbb{Z}^{2 g}$, where the intersection form on $X_{g}$ has block form $\left(\begin{array}{cc}Q_{g} & 0 \\ 0 & 0\end{array}\right)$ with respect to this decomposition for some nondegenerate form $Q_{g}$ on $\mathbb{Z}^{21}$.

Proof. The claim that $H_{1}\left(X_{g}\right)=0$ follows immediately from $X_{g}$ being simply connected, so then $H^{1}\left(X_{g}\right)=0$ as well by the universal coefficient theorem. For $H_{3}\left(X_{g}\right)$, we use the general fact that if $X$ is an orientable $n$-manifold with nonempty boundary, then $H_{n-1}(X)$ injects into $H_{n-1}(X, \partial X) \cong H^{1}(X)$ : the long exact sequence of the pair $(X, \partial X)$ says that

$$
0 \rightarrow H_{n}(X, \partial X) \rightarrow H_{n-1}(\partial X) \rightarrow H_{n-1}(X) \rightarrow H_{n-1}(X, \partial X)
$$

is exact, and the map $H_{n}(X, \partial X) \rightarrow H_{n-1}(\partial X)$ is an isomorphism $\mathbb{Z} \stackrel{\sim}{\rightarrow} \mathbb{Z}$ since it carries the relative fundamental class $[X, \partial X]$ to $[\partial X]$. In general, this implies that $H_{n-1}(X)$ is torsion-free since $H^{1}(X)$ is; in this case, since $H^{1}\left(X_{g}\right)=0$ we have $H_{3}\left(X_{g}\right)=0$ as well.

Since $H_{1}(K 3)=H_{3}(K 3)=0$ and $H_{1}\left(X_{g}\right)=0$, a portion of the Mayer-Vietoris sequence is given by

$$
0 \rightarrow H_{2}\left(Y_{g}\right) \stackrel{i_{2}}{\rightarrow} H_{2}\left(D_{g}\right) \oplus H_{2}\left(X_{g}\right) \stackrel{j}{\rightarrow} H_{2}(K 3) \stackrel{\delta}{\rightarrow} H_{1}\left(Y_{g}\right) \stackrel{i_{1}}{\rightarrow} H_{1}\left(D_{g}\right) \rightarrow 0 .
$$

Now from $H_{1}\left(Y_{g}\right) \cong \mathbb{Z}^{2 g} \oplus \mathbb{Z} /(2 g-2)$ we compute $H_{2}\left(Y_{g}\right) \cong H^{1}\left(Y_{g}\right) \cong \mathbb{Z}^{2 g}$, so that

$$
0 \rightarrow \mathbb{Z}^{2 g} \stackrel{i_{2}}{\rightarrow} \mathbb{Z} \oplus H_{2}\left(X_{g}\right) \stackrel{j}{\rightarrow} \mathbb{Z}^{22} \stackrel{\delta}{\rightarrow} \mathbb{Z}^{2 g} \oplus \mathbb{Z} /(2 g-2) \stackrel{i_{1}}{\rightarrow} \mathbb{Z}^{2 g} \rightarrow 0
$$

is exact. Any torsion in $H_{2}\left(X_{g}\right)$ must lie in $\operatorname{ker}(j)=\operatorname{Im}\left(i_{2}\right)$, and since $\operatorname{Im}\left(i_{2}\right) \cong \mathbb{Z}^{2 g}$ is torsion-free it follows that $H_{2}\left(X_{g}\right)$ is as well, hence $H_{2}\left(X_{g}\right)=\mathbb{Z}^{b_{2}\left(X_{g}\right)}$. The map $i_{1}$ : $\mathbb{Z}^{2 g} \oplus \mathbb{Z} /(2 g-2) \rightarrow \mathbb{Z}^{2 g}$ is surjective and its target $\mathbb{Z}^{2 g}$ is free, so $\operatorname{ker}\left(i_{1}\right)$ must be precisely the torsion subgroup $\mathbb{Z} /(2 g-2)$ of the domain. Thus $\operatorname{Im}(\delta)=\mathbb{Z} /(2 g-2)$, and we deduce from the above sequence that

$$
0 \rightarrow \mathbb{Z}^{2 g} \stackrel{i_{2}}{\rightarrow} \mathbb{Z} \oplus \mathbb{Z}^{b_{2}\left(X_{g}\right)} \stackrel{j}{\rightarrow} \mathbb{Z}^{22} \rightarrow \mathbb{Z} /(2 g-2) \rightarrow 0
$$


is exact. It follows that $\operatorname{Im}(j)$ has index $2 g-2$ and that $b_{2}\left(X_{g}\right)=21+2 g$; the latter fact implies that $H_{2}\left(X_{g}\right)=\mathbb{Z}^{21+2 g}$.

Next, we note that the image of the natural map $H_{2}\left(Y_{g}\right) \rightarrow H_{2}\left(D_{g}\right)$ contributes to $b_{2}^{0}\left(D_{g}\right)$, since any surface inside $Y_{g}$ can be displaced inside a collar neighborhood of $\partial D_{g}$; but $H_{2}\left(D_{g}\right)$ is torsion-free and positive definite, so the map $H_{2}\left(Y_{g}\right) \rightarrow H_{2}\left(D_{g}\right)$ must be zero. Since $i_{2}$ is injective, it follows that $H_{2}\left(Y_{g}\right)=\mathbb{Z}^{2 g}$ injects into $H_{2}\left(X_{g}\right)=\mathbb{Z}^{21+2 g}$. Then $\operatorname{ker}(j) \subset H_{2}\left(X_{g}\right)$ is isomorphic to $\mathbb{Z}^{2 g}$, so that the map $H_{2}\left(X_{g}\right) \rightarrow H_{2}(K 3)$ has rank 21. Since its image is a subgroup of $\mathbb{Z}^{22}$ it is free abelian, hence isomorphic to $\mathbb{Z}^{21}$, and so we have an exact sequence

$$
0 \rightarrow H_{2}\left(Y_{g}\right) \rightarrow H_{2}\left(X_{g}\right) \rightarrow \mathbb{Z}^{21} \rightarrow 0
$$

which splits because $\mathbb{Z}^{21}$ is free. Thus we have a direct sum decomposition

$$
H_{2}\left(X_{g}\right) \cong \mathbb{Z}^{21} \oplus H_{2}\left(Y_{g}\right)
$$

in which the $\mathrm{H}_{2}\left(Y_{g}\right)$ summand lies in the kernel of the intersection form. But we have seen that $b_{2}^{+}\left(X_{g}\right)+b_{2}^{-}\left(X_{g}\right)=21$, so the intersection form must be nondegenerate on the $\mathbb{Z}^{21}$ summand and the proof is complete.

Remark 2.5. The kernel of $j: H_{2}\left(D_{g}\right) \oplus H_{2}\left(X_{g}\right) \rightarrow H_{2}(K 3)$ is the $H_{2}\left(Y_{g}\right) \cong \mathbb{Z}^{2 g}$ summand of $H_{2}\left(X_{g}\right)$, so it restricts to an injective map

$$
j^{\prime}: H_{2}\left(D_{g}\right) \oplus \mathbb{Z}^{21} \rightarrow H_{2}(K 3)
$$

whose domain is the lattice $\mathbb{Z} \oplus \mathbb{Z}^{21}$ with intersection form $\left(\begin{array}{cc}2 g-2 & 0 \\ 0 & Q_{g}\end{array}\right)$ in block form, and $j^{\prime}$ embeds this lattice as an index- $(2 g-2)$ sublattice of $H_{2}(K 3) \cong \mathbb{Z}^{22} \cong 3 H \oplus-2 E_{8}$.

\section{The TOPOLOGY OF EXACT FILLINGS}

We can now use the Calabi-Yau cap $\left(X_{g}, \omega_{g}\right)$ provided by Theorem 2.2 to understand the topology of fillings of $\left(Y_{g}, \xi_{g}\right)$.

Proposition 3.1. Let $(W, \omega)$ be an exact symplectic filling of $\left(Y_{g}, \xi_{g}\right)$. Then the closed symplectic manifold

$$
\left(Z, \omega_{Z}\right)=(W, \omega) \cup_{\left(Y_{g}, \xi_{g}\right)}\left(X_{g}, \omega_{g}\right)
$$

is an integer homology $K$, with $H_{1}(Z ; \mathbb{Z})=H_{3}(Z ; \mathbb{Z})=0$ and $H_{2}(Z ; \mathbb{Z})=\mathbb{Z}^{22}$.

Proof. We can easily verify that $K_{Z} \cdot\left[\omega_{Z}\right]=0$, where $K_{Z}$ is the canonical class of $\left(Z, \omega_{Z}\right)$. Indeed, we can express it as a sum $\left.K_{Z}\right|_{W} \cdot[\omega]+\left.K_{Z}\right|_{X_{g}} \cdot\left[\omega_{g}\right]$, and in the first term we have $[\omega]=0$ since the form $\omega$ is exact, while in the second term we have $\left.K_{Z}\right|_{X_{g}}=0$ because $X_{g}$ has trivial canonical class. Moreover, we have $b_{2}^{+}(Z) \geq b_{2}^{+}\left(X_{g}\right)=2$, with the latter equality provided by Proposition 2.3 .

Since $b_{2}^{+}(Z) \geq 2$ and $K_{Z} \cdot\left[\omega_{Z}\right]=0$, it follows from Taubes [Tau95 that the only SeibergWitten basic classes on $Z$ are $\pm K_{Z}$. Further work of Taubes Tau96] then shows that $K_{Z}=0$, hence 0 is the only basic class: indeed, $K_{Z}$ is Poincaré dual to an embedded symplectic surface $\Sigma$, and we have $K_{Z} \cdot\left[\omega_{Z}\right]=\int_{\Sigma} \omega_{Z} \geq 0$ with equality only if $[\Sigma]=0$. It follows that $Z$ must be symplectically minimal, since otherwise the blow-up formula [FS95. implies that there would be at least two basic classes.

We have now shown that $\left(Z, \omega_{Z}\right)$ is minimal with trivial canonical class, and this proves that its symplectic Kodaira dimension [Li06b] is zero. By work of Morgan-Szabó [MS97, Bauer Bau08, and Li [Li06a, it follows that $Z$ has the rational homology of a K3 surface, an Enriques surface, or a $T^{2}$-bundle over $T^{2}$. The latter two cases imply $b_{2}(Z)=10$ 
and $b_{2}(Z) \leq 6$ respectively, and neither of these can happen - we already know that $b_{2}(Z) \geq b_{2}^{-}(Z) \geq b_{2}^{-}\left(X_{g}\right)=19-$ so $Z$ is a rational homology $\mathrm{K} 3$.

Finally, if $H_{1}(Z)$ is nontrivial, then it is torsion and so the kernel of the abelianization map $\pi_{1}(Z) \rightarrow H_{1}(Z)$ has finite index in $\pi_{1}(Z)$. If the corresponding finite cover $\left(\tilde{Z}, \tilde{\omega}_{Z}\right) \rightarrow$ $\left(Z, \omega_{Z}\right)$ has degree $n=\left|H_{1}(Z)\right|$, then $\left(\tilde{Z}, \tilde{\omega}_{Z}\right)$ has symplectic Kodaira dimension zero and hence signature at least -16 [Bau08, Li06a], whereas $\sigma(\tilde{Z})=-16 n$, so we must have $n=1$ and thus $H_{1}(Z)=0$. It follows from Poincaré duality and the universal coefficient theorem that $H_{3}(Z) \cong H^{1}(Z)=0$, and that $H_{2}(Z) \cong H^{2}(Z)$ is torsion-free since $H_{1}(Z)=0$ is.

Remark 3.2. In the above argument, we see that $Z$ has even intersection form since $K_{Z}=0$ is a characteristic class. If we can show that $\pi_{1}(Z)=1$, then $Z$ will be a simply connected, even, smooth 4-manifold with $b_{2}^{+}(Z)=3$ and $b_{2}^{-}(Z)=19$, implying that it is homotopy equivalent and hence homeomorphic to a K3 surface [Fre82.

For example, if $(W, J)$ is a Stein filling of $\left(Y_{g}, \xi_{g}\right)$ then the inclusion $Y_{g} \hookrightarrow W$ induces a surjection $\pi_{1}\left(Y_{g}\right) \rightarrow \pi_{1}(W)$, and the cap $X_{g}$ is simply connected, so van Kampen's theorem says that $\pi_{1}(Z)=\pi_{1}(W) *_{\pi_{1}\left(Y_{g}\right)} 1=1$. Thus if $(W, J)$ is a Stein filling then $Z$ is homeomorphic to a K3 surface.

Corollary 3.3. If $(W, \omega)$ is an exact symplectic filling of $\left(Y_{g}, \xi_{g}\right)$, then $W$ has the same Betti numbers as the disk cotangent bundle $D T^{*} \Sigma_{g}$, namely $b_{3}(W)=0, b_{2}^{+}(W)=1$ and $b_{2}^{-}(W)=b_{2}^{0}(W)=0$, and $b_{1}(W)=2 g$.

Proof. We glue the cap $\left(X_{g}, \omega_{g}\right)$ to $(W, \omega)$ to form $Z$, which is a homology K3 and thus has signature -16 . Novikov additivity says that $-16=\sigma(W)+\sigma\left(X_{g}\right)$, and from Proposition 2.3 we conclude that $\sigma(W)=1$. In particular, we have $b_{2}^{+}(W) \geq 1$.

Now we consider the Mayer-Vietoris sequence for $Z=W \cup_{Y_{g}} X_{g}$ with coefficients in $\mathbb{Q}$ : since $b_{2}(Z)=22$ and $b_{2}\left(Y_{g}\right)=b_{1}\left(Y_{g}\right)=2 g$, the part of the sequence between $H_{3}(Z ; \mathbb{Q})=0$ and $H_{1}(Z ; \mathbb{Q})=0$ has the form

$$
0 \rightarrow \mathbb{Q}^{2 g} \rightarrow \mathbb{Q}^{b_{2}(W)} \oplus \mathbb{Q}^{21+2 g} \rightarrow \mathbb{Q}^{22} \rightarrow \mathbb{Q}^{2 g} \rightarrow \mathbb{Q}^{b_{1}(W)} \oplus \mathbb{Q}^{0} \rightarrow 0 .
$$

Since we already know that $b_{2}(W) \geq 1$, an easy exercise shows that in fact $b_{2}(W)=1$ and $b_{1}(W)=2 g$; then $\sigma(W)=1$ implies that $b_{2}^{+}(W)=1$ and $b_{2}^{-}(W)=b_{2}^{0}(W)=0$ as claimed. Similarly, between $H_{4}(W ; \mathbb{Q}) \oplus H_{4}\left(X_{g} ; \mathbb{Q}\right)=0$ and $H_{3}(Z ; \mathbb{Q})=0$, we have

$$
0 \rightarrow \mathbb{Q} \rightarrow \mathbb{Q} \rightarrow \mathbb{Q}^{b_{3}(W)} \oplus \mathbb{Q}^{0} \rightarrow 0
$$

and this can only be exact if $b_{3}(W)=0$.

Theorem 3.4. If $(W, \omega)$ is an exact filling of $\left(Y_{g}, \xi_{g}\right)$, then for some integer $d$ such that $d^{2}$ divides $g-1$ we have $H_{3}(W ; \mathbb{Z})=0 ; H_{2}(W ; \mathbb{Z}) \cong \mathbb{Z}$, with intersection form $\left\langle\frac{2 g-2}{d^{2}}\right\rangle ;$ and $H_{1}(W ; \mathbb{Z}) \cong \mathbb{Z}^{2 g} \oplus \mathbb{Z} / d \mathbb{Z}$.

Proof. We recall from the proof of Proposition 2.4 that $H_{3}(W)$ is torsion-free, and so $b_{3}(W)=0$ implies that $H_{3}(W)=0$.

Now we write $Z=W \cup_{Y_{g}} X_{g}$, with $Z$ an integer homology $\mathrm{K} 3$, and consider the MayerVietoris sequence over $\mathbb{Z}$ :

$$
0 \rightarrow H_{2}\left(Y_{g}\right) \stackrel{i}{\rightarrow} H_{2}(W) \oplus H_{2}\left(X_{g}\right) \stackrel{j}{\rightarrow} H_{2}(Z) \stackrel{\delta}{\rightarrow} H_{1}\left(Y_{g}\right) .
$$

We know that $H_{1}\left(Y_{g}\right)=\mathbb{Z}^{2 g} \oplus \mathbb{Z} /(2 g-2)$, hence $H_{2}\left(Y_{g}\right)=H^{1}\left(Y_{g}\right)=\mathbb{Z}^{2 g}$, and similarly we know the homology of $X_{g}$ from Proposition 2.4. Any torsion in $H_{2}(W)$ must lie in 
$\operatorname{ker}(j)=\operatorname{Im}(i)$ since $H_{2}(Z)=\mathbb{Z}^{22}$ is free, but $\operatorname{Im}(i) \cong \mathbb{Z}^{2 g}$ is also free, so $H_{2}(W)$ is torsionfree and thus $H_{2}(W)=\mathbb{Z}$ by Corollary 3.3 .

Since $H_{2}(W)$ is positive definite and both $H_{2}\left(Y_{g}\right)$ and $H_{2}(W)$ are torsion-free, the map $H_{2}\left(Y_{g}\right) \rightarrow H_{2}(W)$ is zero, and we know that $H_{2}\left(X_{g}\right)$ decomposes as $\mathbb{Z}^{21} \oplus H_{2}\left(Y_{g}\right)$. Thus we can split off the $H_{2}\left(Y_{g}\right) \stackrel{\sim}{\rightarrow} H_{2}\left(Y_{g}\right)$ component of $i$ in the above sequence, leaving us with

$$
0 \rightarrow \mathbb{Z} \oplus \mathbb{Z}^{21} \rightarrow H_{2}(Z) \stackrel{\delta}{\rightarrow} H_{1}\left(Y_{g}\right) .
$$

Let $\Lambda \subset H_{2}(Z)$ be the image of $\mathbb{Z} \oplus \mathbb{Z}^{21}$; then $\Lambda$ is a sublattice of rank 22, so it has finite index, which must equal $|\operatorname{Im}(\delta)|$. But from $H_{1}\left(Y_{g}\right)=\mathbb{Z}^{2 g} \oplus \mathbb{Z} /(2 g-2)$ it follows that $\operatorname{Im}(\delta)$ is a subgroup of $\mathbb{Z} /(2 g-2)$, which then has order $\frac{2 g-2}{d}$ for some integer $d \geq 1$. Since $H_{1}\left(X_{g}\right)=H_{1}(Z)=0$, the portion $H_{2}(Z) \stackrel{\delta}{\rightarrow} H_{1}\left(Y_{g}\right) \rightarrow H_{1}(W) \rightarrow 0$ of the sequence shows that $H_{1}(W)$ is isomorphic to $H_{1}\left(Y_{g}\right) / \operatorname{Im}(\delta) \cong \mathbb{Z}^{2 g} \oplus \mathbb{Z} / d$.

Let $e_{1}, \ldots, e_{22}$ be an integral basis of $\Lambda$, where $e_{1}$ generates the direct summand $H_{2}(W) \cong$ $\mathbb{Z}$ and $e_{2}, \ldots, e_{22}$ is an integral basis of $\mathbb{Z}^{21} \subset H_{2}\left(X_{g}\right)$, and form a matrix $A$ whose columns are the elements $e_{1}, \ldots, e_{22}$ expressed in an integral basis of $H_{2}(Z) \cong \mathbb{Z}^{22}$. Letting $Q_{Z}$ be the intersection form on $H_{2}(Z)$ in this latter basis, then, the intersection form on $\Lambda$ in the basis $\left\{e_{i}\right\}$ is given by $Q_{\Lambda}=A^{T} Q_{Z} A$, and we have $\operatorname{det}\left(Q_{\Lambda}\right)= \pm\left(\frac{2 g-2}{d}\right)^{2}$ since $Q_{Z}$ is unimodular and $|\operatorname{det}(A)|=\left[H_{2}(Z): \Lambda\right]=\frac{2 g-2}{d}$.

On the other hand, we can write $Q_{\Lambda}$ in block form with respect to this basis as $\left(\begin{array}{cc}e_{1} \cdot e_{1} & 0 \\ 0 & Q_{g}\end{array}\right)$, where $Q_{g}$ is the nondegenerate intersection form on $\mathbb{Z}^{21} \subset H_{2}\left(X_{g}\right)$; note that $\left|\operatorname{det}\left(Q_{g}\right)\right|$ does not depend on $W$ but only on the cap $X_{g}$. From this it is clear that $\operatorname{det}\left(Q_{\Lambda}\right)=$ $\left(e_{1} \cdot e_{1}\right) \operatorname{det}\left(Q_{g}\right)$, so it follows that $\left(e_{1} \cdot e_{1}\right)\left|\operatorname{det}\left(Q_{g}\right)\right|=\left(\frac{2 g-2}{d}\right)^{2}$. In the case where $W$ is the disk cotangent bundle $D T^{*} \Sigma_{g}$ we have $e_{1} \cdot e_{1}=\Sigma_{g}^{2}=2 g-2$ and $d=1$ (see Remark 2.5), so it follows that $\left|\operatorname{det}\left(Q_{g}\right)\right|$ is equal to $2 g-2$. We conclude that

$$
e_{1} \cdot e_{1}=\frac{\left(\frac{2 g-2}{d}\right)^{2}}{2 g-2}=\frac{2 g-2}{d^{2}} .
$$

Since $Z$ is a homology $\mathrm{K} 3$ it has an even intersection form, so $e_{1} \cdot e_{1}$ must be an even integer and we have $d^{2} \mid g-1$, completing the proof.

Corollary 3.5. If $g-1$ is square-free then any exact filling of $\left(Y_{g}, \xi_{g}\right)$ has the same homology and intersection form as the disk cotangent bundle $D T^{*} \Sigma_{g}$.

\section{The topology of Stein fillings}

4.1. The homology of a Stein filling. In this section we further investigate the topology of a filling $(W, \omega)$ of $\left(Y_{g}, \xi_{g}\right)$ which is not only exact but Stein; in this case we denote it by $(W, J)$ to avoid confusion. In this case $W$ has a handle decomposition consisting of only 0-, 1-, and 2-handles, from which it follows classically that the inclusion $i: Y_{g} \hookrightarrow W$ induces a surjection $\pi_{1}\left(Y_{g}\right) \stackrel{i_{*}}{\rightarrow} \pi_{1}(W)$. We note that since $Y_{g}$ is a circle bundle over $\Sigma_{g}$ with Euler number $2 g-2$, its fundamental group has presentation

$$
\pi_{1}\left(Y_{g}\right)=\left\langle a_{1}, \ldots, a_{g}, b_{1}, \ldots, b_{g}, t \mid \prod_{i=1}^{g}\left[a_{i}, b_{i}\right]=t^{2 g-2},\left[a_{i}, t\right]=\left[b_{i}, t\right]=1\right\rangle,
$$


where $t$ represents a circle fiber and is central. We will define $2 g+1$ distinguished elements of $\pi_{1}(W)$ by

$$
\alpha_{j}=i_{*}\left(a_{j}\right), \quad \quad \beta_{j}=i_{*}\left(b_{j}\right), \quad \tau=i_{*}(t)
$$

for $j=1, \ldots, g$. Since $i_{*}$ is surjective, we know that $\tau$ is central and that these $2 g+1$ elements generate $\pi_{1}(W)$; in fact, it turns out that $\alpha_{1}, \ldots, \alpha_{g}$ and $\beta_{1}, \ldots, \beta_{g}$ suffice.

Proposition 4.1. Suppose that $(W, J)$ is a Stein filling of $\left(Y_{g}, \xi_{g}\right)$ as above, and let $H \subset$ $\pi_{1}\left(Y_{g}\right)$ denote the subgroup generated by $a_{1}, \ldots, a_{g}, b_{1}, \ldots, b_{g}$. If $i_{*}: \pi_{1}\left(Y_{g}\right) \rightarrow \pi_{1}(W)$ is the inclusion-induced map, then $\left.i_{*}\right|_{H}$ is surjective; in other words, $i_{*}(H)=\pi_{1}(W)$, and so $\pi_{1}(W)$ is generated by the elements $\alpha_{1}, \ldots, \alpha_{g}$ and $\beta_{1}, \ldots, \beta_{g}$.

Proof. It is not hard to check that $H$ is normal of index $2 g-2$, since the only other generator in the above presentation is central (namely $t$ ) and the quotient $\pi_{1}\left(Y_{g}\right) / H$ is $\left\langle t \mid t^{2 g-2}=1\right\rangle$. Since $i_{*}$ is surjective, it is also easy to see that $i_{*}(H)$ is a normal subgroup of $\pi_{1}(W)$. Moreover, $i_{*}$ induces a map

$$
\pi_{1}\left(Y_{g}\right) / H \rightarrow \pi_{1}(W) / i_{*}(H)
$$

between the respective quotients, and this map is surjective, so since $\pi_{1}\left(Y_{g}\right) / H$ is a finite cyclic group generated by $[t]$ it follows that $\pi_{1}(W) / i_{*}(H)$ is also a finite cyclic group which is generated by $[\tau]$. Thus $i_{*}(H)$ is a normal subgroup of $\pi_{1}(W)$ of some finite index $k \geq 1$ which divides $\left|\pi_{1}\left(Y_{g}\right) / H\right|=2 g-2$.

Let $p: \tilde{W} \rightarrow W$ be a finite $k$-fold covering such that $p_{*}\left(\pi_{1}(\tilde{W})\right)=i_{*}(H)$. Then $\tilde{W}$ is also a Stein domain, and its boundary $\tilde{Y}=\partial \tilde{W}$ is a $k$-fold cover of $Y_{g}=\partial W$, which must be connected since Stein domains have connected boundary. Thus $G=\left(\left.p\right|_{\tilde{Y}}\right)_{*}\left(\pi_{1}(\tilde{Y})\right)$ is an index- $k$ subgroup of $\pi_{1}\left(Y_{g}\right)$. The cover $\tilde{Y} \rightarrow Y_{g}$ is normal, implying that $G$ is moreover a normal subgroup of $\pi_{1}\left(Y_{g}\right)$ : indeed, the cover $\tilde{W} \rightarrow W$ is normal since $i_{*}(H)$ is a normal subgroup of $\pi_{1}(W)$, so its deck transformations act transitively on each fiber, and these restrict to deck transformations of $\tilde{Y}$, so the latter act transitively on fibers of $\tilde{Y}$.

We now consider the commutative diagram

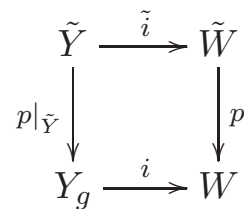

where $i$ and $\tilde{i}$ are the respective inclusion maps of each manifold into the Stein domain which it bounds, and thus induce surjections on the respective fundamental groups. We have $p_{*}\left(\pi_{1}(\tilde{W})\right)=i_{*}(H)$ by construction, and since $\tilde{i}_{*}\left(\pi_{1}(\tilde{Y})\right)=\pi_{1}(\tilde{W})$ we can write

$$
i_{*}(H)=p_{*}\left(\tilde{i}_{*}\left(\pi_{1}(\tilde{Y})\right)\right)=i_{*}\left(\left(\left.p\right|_{\tilde{Y}}\right)_{*}\left(\pi_{1}(\tilde{Y})\right)\right)=i_{*}(G) .
$$

Thus if $t^{j} \in G$ for some $j$, then we have $\tau^{j} \in i_{*}(G)=i_{*}(H)$, and so $k$ divides $j$.

Now we consider the composition $\varphi: \tilde{Y} \stackrel{p}{\rightarrow} Y_{g} \rightarrow \Sigma_{g}$, where we are now using $p$ to denote the restriction $\left.p\right|_{\tilde{Y}}$. The preimage of a point $x \in \Sigma_{g}$ is a $k$-fold cover of the circle fiber above $x$ in $Y_{g}$, which we identify with $t$ (at least up to conjugation, since we should pick a base point). If this preimage is disconnected, then one of its components is a circle $\gamma \subset \tilde{Y}$ which is an $l$-fold cover of the circle fiber in $Y_{g}$ for some $1 \leq l<k$. Thus $p_{*}(\gamma)$ is conjugate to $t^{l}$; but $G$ is normal and does not contain $t^{l}$, so it cannot actually contain $p_{*}(\gamma)$ either. We 
conclude that $\varphi^{-1}(x)$ is a circle, and hence that $\tilde{Y}$ is also a circle bundle over $\Sigma_{g}$. Its Euler number is then $\frac{2 g-2}{k}$, though we only need that it is nonzero: if it were zero, then the image under $p$ of a section would give a section of $Y_{g}$, which has nonzero Euler number.

From the above we see that $b_{1}(\tilde{Y})=2 g$, and since $H_{1}(\tilde{Y})$ surjects onto $H_{1}(\tilde{W})$ it follows that $b_{1}(\tilde{W}) \leq 2 g$, hence

$$
\chi(\tilde{W})=1-b_{1}(\tilde{W})+b_{2}(\tilde{W}) \geq 1-2 g .
$$

But we also know that $\chi(\tilde{W})=k \chi(W)=k(2-2 g)$, so we have $k(2-2 g) \geq 1-2 g$, or equivalently $(k-1)(2-2 g) \geq-1$. Since $2-2 g \leq-2$, this can only hold if $k=1$; but $k$ is the index of $i_{*}(H)$ in $\pi_{1}(W)$, so the two must be equal.

Theorem 4.2. Let $(W, J)$ be a Stein filling of $\left(Y_{g}, \xi_{g}\right)$. Then $W$ has the same integral homology and intersection form as the disk cotangent bundle $D T^{*} \Sigma_{g}$. In particular, we have $H_{1}(W) \cong \mathbb{Z}^{2 g}$, and the intersection form on $H_{2}(W) \cong \mathbb{Z}$ is $\langle 2 g-2\rangle$.

Proof. In light of Theorem 3.4 we know that $H_{1}(W) \cong \mathbb{Z}^{2 g} \oplus \mathbb{Z} / d \mathbb{Z}$ for some $d \geq 1$, and that it suffices to show that $d=1$. Now according to Proposition 4.1, the fundamental group $\pi_{1}(W)$ is generated by the $2 g$ elements $\alpha_{1}, \ldots, \alpha_{g}, \beta_{1}, \ldots, \beta_{g}$, hence its abelianization $H_{1}(W)$ is also generated by the corresponding homology classes. However, if $d>1$ then any presentation of $\mathbb{Z}^{2 g} \oplus \mathbb{Z} / d \mathbb{Z}$ requires at least $2 g+1$ generators, so we must have $d=1$.

Theorem 4.2 tells us the first group homology of $\pi_{1}(W)$, since $H_{1}\left(\pi_{1}(W) ; \mathbb{Z}\right)=H_{1}(W ; \mathbb{Z})$. The second homology of $\pi_{1}(W)$ will also be useful later:

Proposition 4.3. If $(W, J)$ is a Stein filling of $\left(Y_{g}, \xi_{g}\right)$, then $H_{2}\left(\pi_{1}(W) ; \mathbb{Z}\right) \cong \mathbb{Z}$.

Proof. Let $\pi=\pi_{1}(W)$, and recall that $H_{2}(W) \cong \mathbb{Z}$. The group homology $H_{2}(\pi ; \mathbb{Z})=$ $H_{2}(K(\pi, 1) ; \mathbb{Z})$ is classically known to be isomorphic to the cokernel of the Hurewicz map

$$
h: \pi_{2}(W) \rightarrow H_{2}(W),
$$

which is $\mathbb{Z} / \operatorname{Im}(h)$, so $H_{2}(\pi ; \mathbb{Z})$ is $\mathbb{Z}$ if the Hurewicz map is zero and finite otherwise. The $2 g$ elements $\alpha_{1}, \ldots, \alpha_{g}, \beta_{1}, \ldots, \beta_{g}$ generate $\pi$ by Proposition 4.1, so their images generate $H_{1}(W ; \mathbb{Z})=\mathbb{Z}^{2 g}$ and are thus linearly independent over $\mathbb{Q}$.

Supposing that $h$ is nonzero, we have $H_{2}(\pi ; \mathbb{Q})=0$. According to Stallings Sta65, Theorem 7.4], the linear independence of the $\alpha_{i}$ and $\beta_{i}$ in $H_{1}(\pi)$ and the vanishing of $H_{2}(\pi ; \mathbb{Q})$ guarantee that $\alpha_{1}, \ldots, \alpha_{g}, \beta_{1}, \ldots, \beta_{g}$ form a basis of a free subgroup of $\pi$, and we conclude that $\pi$ is the free group $F_{2 g}$. The element $\prod_{j=1}^{g}\left[\alpha_{j}, \beta_{j}\right]$ of $\pi$ is central, since it equals $\tau^{2 g-2}$ and $\tau$ is central; but free groups have trivial center, so $\prod_{j=1}^{g}\left[\alpha_{j}, \beta_{j}\right]=1$ and thus $\pi$ is a nontrivial quotient of $F_{2 g}$. Since finitely generated free groups are Hopfian, a nontrivial quotient of $F_{2 g}$ cannot be isomorphic to $F_{2 g}$, and we have a contradiction.

Since the class $[t]$ of the circle fiber generates the torsion summand of $H_{1}\left(Y_{g}\right)$, and Theorem 4.2 says that $H_{1}(W)$ is torsion-free, we see that $[\tau]=0$ in $H_{1}(W)$. Thus $\tau$ lies in the commutator subgroup of $\pi_{1}(W)$. In Section 4.2 we will see that in fact $\tau=1$ in $\pi_{1}(W)$.

4.2. The fundamental group of a Stein filling. Let $(W, J)$ denote a Stein filling of $\left(Y_{g}, \xi_{g}\right)$ as usual. Our goal in this section is to explicitly determine its fundamental group:

Theorem 4.4. The fundamental group $\pi_{1}(W)$ is isomorphic to $\pi_{1}\left(\Sigma_{g}\right)$. 
Our strategy will be to first show that $\pi_{1}(W)$ must be an extension of $\pi_{1}\left(\Sigma_{g}\right)$ by a cyclic group and then use what we know about its group homology to show that this cyclic group is in fact trivial.

Summarizing what we know so far about $\pi_{1}(W)$, we have seen that it is a quotient of

$$
\left\langle\alpha_{1}, \ldots, \alpha_{g}, \beta_{1}, \ldots, \beta_{g}, \tau \mid \prod_{i=1}^{g}\left[\alpha_{i}, \beta_{i}\right]=\tau^{2 g-2},\left[\alpha_{i}, \tau\right]=\left[\beta_{i}, \tau\right]=1\right\rangle,
$$

where the central element $\tau$ is the image of a circle fiber $t \in \pi_{1}\left(Y_{g}\right)$. Moreover, $H_{1}(W ; \mathbb{Z})=$ $\mathbb{Z}^{2 g}$ is generated by the elements $\alpha_{i}$ and $\beta_{i}$, and the central element $\tau$ belongs to the commutator subgroup of $\pi_{1}(W)$. Thus there is a surjection

$$
p: \pi_{1}\left(\Sigma_{g}\right)=\left\langle\alpha_{1}, \ldots, \alpha_{g}, \beta_{1}, \ldots, \beta_{g} \mid \prod_{i=1}^{g}\left[\alpha_{i}, \beta_{i}\right]=1\right\rangle \rightarrow \pi_{1}(W) /\langle\tau\rangle
$$

through which the abelianization map ab $: \pi_{1}\left(\Sigma_{g}\right) \rightarrow \mathbb{Z}^{2 g}$ factors as

$$
\pi_{1}\left(\Sigma_{g}\right) \stackrel{p}{\rightarrow} \pi_{1}(W) /\langle\tau\rangle \stackrel{\mathrm{ab}}{\rightarrow} \mathbb{Z}^{2 g} .
$$

Such a factorization exists for any surjection $p: \pi_{1}\left(\Sigma_{g}\right) \rightarrow \pi_{1}(W) /\langle\tau\rangle$ : since $\pi_{1}\left(\Sigma_{g}\right) \stackrel{\text { abop }}{\longrightarrow}$ $\mathbb{Z}^{2 g}$ is a map to an abelian group, it factors as $\pi_{1}\left(\Sigma_{g}\right) \stackrel{\text { ab }}{\longrightarrow} \mathbb{Z}^{2 g} \stackrel{\psi}{\rightarrow} \mathbb{Z}^{2 g}$, and $\psi$ is a surjection $\mathbb{Z}^{2 g} \rightarrow \mathbb{Z}^{2 g}$ since ab o $p$ is onto, so it is an isomorphism and then ab : $\pi_{1}\left(\Sigma_{g}\right) \rightarrow \mathbb{Z}^{2 g}$ is equal to $\left(\psi^{-1} \circ \mathrm{ab}\right) \circ p$.

If we fix a surjection $\varphi: \mathbb{Z}^{2 g} \rightarrow \mathbb{Z} / n \mathbb{Z}$ for some $n>1$, then we have a collection of surjective maps of the form

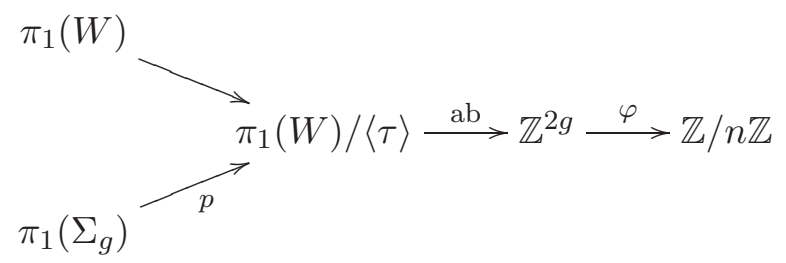

and the kernels of the maps $\pi_{1}(W) \rightarrow \mathbb{Z} / n \mathbb{Z}$ and $\pi_{1}\left(\Sigma_{g}\right) \rightarrow \mathbb{Z} / n \mathbb{Z}$ define normal, $n$-fold cyclic covers $W^{\prime}$ and $\Sigma_{g^{\prime}}$ of $W$ and $\Sigma_{g}$ respectively, where $2-2 g^{\prime}=n(2-2 g)$.

Definition 4.5. Let $(W, J)$ be a Stein filling of $\left(Y_{g}, \xi_{g}\right)$, and let $p: \pi_{1}\left(\Sigma_{g}\right) \rightarrow \pi_{1}(W) /\langle\tau\rangle$ be a surjection. If $\Sigma_{g^{\prime}} \rightarrow \Sigma_{g}$ and $W^{\prime} \rightarrow W$ are the finite cyclic covers produced by the above construction, then we will say that $\left(\Sigma_{g^{\prime}}, W^{\prime}\right)$ is induced by $(p, \varphi)$.

Since $W^{\prime}$ is a finite cover of a Stein manifold it has a natural Stein structure $J^{\prime}$, so its boundary $\left(Y^{\prime}, \xi^{\prime}\right)$ is connected, and as in the proof of Proposition 4.1 it follows that $Y^{\prime}$ is a normal, $n$-fold cyclic cover of $Y_{g}$.

Lemma 4.6. If $\left(\Sigma_{g^{\prime}}, W^{\prime}\right)$ is induced by $(p, \varphi)$, then $\left(W^{\prime}, J^{\prime}\right)$ is a Stein filling of the canonical contact structure $\left(Y^{\prime}, \xi^{\prime}\right)=\left(Y_{g^{\prime}}, \xi_{g^{\prime}}\right)$ on the unit cotangent bundle of $\Sigma_{g^{\prime}}$.

Proof. The circle fiber $t \in \pi_{1}\left(Y_{g}\right)$ is in the kernel of $\pi_{1}\left(Y_{g}\right) \stackrel{i_{*}}{\rightarrow} \pi_{1}(W) \rightarrow \mathbb{Z} / n \mathbb{Z}$ since it maps to $\tau \in \pi_{1}(W)$, so it lifts to a closed curve in $Y^{\prime}$. Its preimage in $Y^{\prime}$ therefore consists of $n$ disjoint circles, so the orbit space $Y^{\prime} / S^{1}$ is an $n$-fold cover of $\Sigma_{g}$, hence $Y^{\prime}$ is a circle bundle over $\Sigma_{g^{\prime}}$. The Euler class of $Y^{\prime} \rightarrow \Sigma_{g^{\prime}}$ is then $n$ times the Euler class of $Y_{g} \rightarrow \Sigma_{g}$, namely $-n \chi\left(\Sigma_{g}\right)=-\chi\left(\Sigma_{g^{\prime}}\right)$, so in fact $Y^{\prime}$ is the unit cotangent bundle $Y_{g^{\prime}}$ of $\Sigma_{g^{\prime}}$. 
Since the contact structure $\xi_{g}$ is tangent to the fibers of $Y_{g} \rightarrow \Sigma_{g}$, its cover $\xi^{\prime}$ is likewise tangent to the fibers of $Y_{g^{\prime}} \rightarrow \Sigma_{g^{\prime}}$, and the only contact structure on the unit cotangent bundle of $\Sigma_{g^{\prime}}$ with Legendrian fibers is the canonical one Gir01, Proposition 3.3] (cf. also [Lut83]. Thus $\left(Y^{\prime}, \xi^{\prime}\right)=\left(Y_{g^{\prime}}, \xi_{g^{\prime}}\right)$, and so $\left(W^{\prime}, J^{\prime}\right)$ is a Stein filling of $\left(Y_{g^{\prime}}, \xi_{g^{\prime}}\right)$.

Proposition 4.7. Suppose that $\left(\Sigma_{g^{\prime}}, W^{\prime}\right)$ is induced by $(p, \varphi)$. Identifying $\pi_{1}\left(\Sigma_{g^{\prime}}\right)$ as a subgroup of $\pi_{1}\left(\Sigma_{g}\right)$, the map $p$ induces a surjection

$$
p^{\prime}: \pi_{1}\left(\Sigma_{g^{\prime}}\right) \rightarrow \pi_{1}\left(W^{\prime}\right) /\left\langle\tau^{\prime}\right\rangle
$$

such that $\operatorname{ker}\left(p^{\prime}\right)=\operatorname{ker}(p)$.

Proof. It is clear that $\langle\tau\rangle \subset \pi_{1}\left(W^{\prime}\right)$, viewing the latter as a subgroup of $\pi_{1}(W)$, since $\tau$ is in the kernel of $\pi_{1}(W) \rightarrow \mathbb{Z} / n \mathbb{Z}$. Moreover, if $\tau^{\prime} \in \pi_{1}\left(W^{\prime}\right)$ denotes the image of the circle fiber $t^{\prime} \in \pi_{1}\left(Y_{g^{\prime}}\right)$, then since $t^{\prime}$ projects to the circle fiber $t \in \pi_{1}\left(Y_{g}\right)$, the covering map $W^{\prime} \rightarrow W$ sends $\tau^{\prime}$ to $\tau$, so we have

$$
\langle\tau\rangle \cap \pi_{1}\left(W^{\prime}\right)=\left\langle\tau^{\prime}\right\rangle
$$

Thus the kernel of $\pi_{1}\left(W^{\prime}\right) \hookrightarrow \pi_{1}(W) \rightarrow \pi_{1}(W) /\langle\tau\rangle$ is $\left\langle\tau^{\prime}\right\rangle$, inducing an injective map

$$
\frac{\pi_{1}\left(W^{\prime}\right)}{\left\langle\tau^{\prime}\right\rangle} \hookrightarrow \frac{\pi_{1}(W)}{\langle\tau\rangle}
$$

and it follows that $\pi_{1}\left(W^{\prime}\right) /\left\langle\tau^{\prime}\right\rangle$ has index $n$ in $\pi_{1}(W) /\langle\tau\rangle$. Since $\pi_{1}\left(W^{\prime}\right)$ is by definition the kernel of the map $\pi_{1}(W) \rightarrow \mathbb{Z} / n \mathbb{Z}$, the group $\pi_{1}\left(W^{\prime}\right) /\left\langle\tau^{\prime}\right\rangle$ sits in the kernel of the surjective $\pi_{1}(W) /\langle\tau\rangle \stackrel{\varphi \circ a b}{\longrightarrow} \mathbb{Z} / n \mathbb{Z}$, and this kernel has index $n$ so we conclude that

$$
\pi_{1}\left(W^{\prime}\right) /\left\langle\tau^{\prime}\right\rangle=\operatorname{ker}\left(\varphi \circ \mathrm{ab}: \pi_{1}(W) /\langle\tau\rangle \rightarrow \mathbb{Z} / n \mathbb{Z}\right) .
$$

Since $\pi_{1}\left(\Sigma_{g^{\prime}}\right)$ is the kernel of $(\varphi \circ \mathrm{ab}) \circ p$, it follows that $p\left(\pi_{1}\left(\Sigma_{g^{\prime}}\right)\right)$ lies in $\operatorname{ker}(\varphi \circ \mathrm{ab})$, and so $p$ restricts to a map

$$
p^{\prime}: \pi_{1}\left(\Sigma_{g^{\prime}}\right) \rightarrow \pi_{1}\left(W^{\prime}\right) /\left\langle\tau^{\prime}\right\rangle,
$$

which is easily seen to be surjective just as $p$ is. Finally, since $p^{\prime}$ is the restriction of $p$ to $\pi_{1}\left(\Sigma_{g^{\prime}}\right) \subset \pi_{1}\left(\Sigma_{g}\right)$ it follows that $\operatorname{ker}\left(p^{\prime}\right)=\operatorname{ker}(p) \cap \pi_{1}\left(\Sigma_{g^{\prime}}\right)$. But $\operatorname{ker}(p) \subset \operatorname{ker}(\varphi \circ$ ab $\circ p)=$ $\pi_{1}\left(\Sigma_{g^{\prime}}\right)$, and so $\operatorname{ker}(p)=\operatorname{ker}\left(p^{\prime}\right)$ as claimed.

Proposition 4.7 allows us to characterize $\pi_{1}(W)$ as a cyclic extension of a surface group:

Proposition 4.8. The fundamental group $\pi_{1}(W)$ is a central extension of $\pi_{1}\left(\Sigma_{g}\right)$ by a cyclic group. More precisely, there is a short exact sequence

$$
1 \rightarrow\langle\tau\rangle \rightarrow \pi_{1}(W) \rightarrow \pi_{1}\left(\Sigma_{g}\right) \rightarrow 1
$$

with the image of $\langle\tau\rangle$ being central in $\pi_{1}(W)$.

Proof. It suffices to show that the surjection $p: \pi_{1}\left(\Sigma_{g}\right) \rightarrow \pi_{1}(W) /\langle\tau\rangle$ is also injective. Supposing otherwise, let $x$ be a nontrivial element of $\operatorname{ker}(p)$. Since surface groups are RFRS Ago08 (cf. also Hem72]), there is a descending chain of subgroups

$$
\pi_{1}\left(\Sigma_{g}\right)=G_{0} \supset G_{1} \supset G_{2} \supset \ldots
$$

such that each $G_{i+1}$ is a normal subgroup of $G_{i}$ with finite cyclic quotient, defined as the kernel of a map which factors through $G_{i} \rightarrow\left(G_{i}\right)^{\text {ab }}$, and $\bigcap_{i=0}^{\infty} G_{i}=\{1\}$. This corresponds to a tower of normal, finite cyclic covers

$$
\cdots \rightarrow \Sigma_{g_{2}} \rightarrow \Sigma_{g_{1}} \rightarrow \Sigma_{g_{0}}=\Sigma_{g}
$$


such that $\pi_{1}\left(\Sigma_{g_{i+1}}\right)=\operatorname{ker}\left(\pi_{1}\left(\Sigma_{g_{i}}\right) \stackrel{\text { ab }}{\longrightarrow} \mathbb{Z}^{2 g_{i}} \stackrel{\varphi_{i}}{\longrightarrow} \mathbb{Z} / n_{i} \mathbb{Z}\right)$ for some $\varphi_{i}$. Now by induction, since ab $: \pi_{1}\left(\Sigma_{g}\right) \rightarrow \mathbb{Z}^{2 g}$ factors through $p_{0}=p: \pi_{1}\left(\Sigma_{g}\right) \rightarrow \pi_{1}\left(W_{0}\right) /\left\langle\tau_{0}\right\rangle$, with $W_{0}=W$, we can construct for each $i \geq 0$ a normal cyclic cover $\left(W_{i+1}, J_{i+1}\right)$ of $\left(W_{i}, J_{i}\right)$ as above, with $\left(\Sigma_{g_{i+1}}, W_{i+1}\right)$ induced by $\left(p_{i}, \varphi_{i}\right)$. By Lemma 4.6. $\left(W_{i+1}, J_{i+1}\right)$ is a Stein filling of $\left(Y_{g_{i+1}}, \xi_{g_{i+1}}\right)$, and Proposition 4.7 provides a surjection

$$
p_{i+1}: \pi_{1}\left(\Sigma_{g_{i+1}}\right) \rightarrow \pi_{1}\left(W_{i+1}\right) /\left\langle\tau_{i+1}\right\rangle
$$

with $\operatorname{ker}\left(p_{i+1}\right)=\operatorname{ker}\left(p_{i}\right)$. Thus $x \in \operatorname{ker}\left(p_{0}\right)$ implies that $x \in \operatorname{ker}\left(p_{i}\right) \subset G_{i}$ for all $i$. But since $\bigcap G_{i}=\{1\}$ it follows that $x \notin G_{k}$ for some $k \geq 0$, and this is a contradiction.

Proof of Theorem 4.4. The circle fiber $\tau$ generates a $\mathbb{Z} / n \mathbb{Z}$ subgroup for some $n \geq 0$ (with $n=0$ if it is nontorsion), so Proposition 4.8 provides a short exact sequence of groups

$$
1 \rightarrow \mathbb{Z} / n \mathbb{Z} \rightarrow \pi_{1}(W) \rightarrow \pi_{1}\left(\Sigma_{g}\right) \rightarrow 1
$$

for some $n \geq 0$. We will show that $n=1$, and thus that $\pi_{1}(W) \rightarrow \pi_{1}\left(\Sigma_{g}\right)$ is an isomorphism.

The homologies of these groups with $\mathbb{Z}$ coefficients are related by the Lyndon/HochschildSerre spectral sequence (see e.g. [Bro82]),

$$
E_{p, q}^{2}=H_{p}\left(\pi_{1}\left(\Sigma_{g}\right) ; H_{q}(\mathbb{Z} / n \mathbb{Z} ; \mathbb{Z})\right) \quad \Longrightarrow \quad H_{p+q}\left(\pi_{1}(W) ; \mathbb{Z}\right) .
$$

Since $\pi_{1}\left(\Sigma_{g}\right)$ has cohomological dimension 2 , the $E^{2}$ page is supported in the interval $0 \leq p \leq 2$. Moreover, the homology of $\mathbb{Z} / n \mathbb{Z}$ is given by (letting $k \geq 1$ ):

$$
H_{q}(\mathbb{Z} ; \mathbb{Z})=\left\{\begin{array}{ll}
\mathbb{Z}, & q=0,1 \\
0, & q \geq 2,
\end{array} \quad H_{q}(\mathbb{Z} / k \mathbb{Z} ; \mathbb{Z})= \begin{cases}\mathbb{Z}, & q=0 \\
\mathbb{Z} / k \mathbb{Z}, & q \text { odd } \\
0, & q \geq 2 \text { even }\end{cases}\right.
$$

In either case, the differential $d^{2}: E_{p, q}^{2} \rightarrow E_{p-2, q+1}^{2}$ must be identically zero with the possible exception of the map $\delta: E_{2,0}^{2} \rightarrow E_{0,1}^{2}$, since otherwise either the source or the target vanishes. Each of the higher differentials $d^{r}: E_{p, q}^{r} \rightarrow E_{p-r, q+r-1}^{r}$ must vanish for $r \geq 3$ because either $p>2$ or $p-r<0$, so the spectral sequence collapses at the $E^{3}$ page, and we have

$$
E_{0,2}^{\infty}=0, \quad E_{1,1}^{\infty}=(\mathbb{Z} / n \mathbb{Z})^{2 g}, \quad E_{2,0}^{\infty}=\operatorname{ker}(\delta: \mathbb{Z} \rightarrow \mathbb{Z} / n \mathbb{Z}) .
$$

The convergence of this spectral sequence means that these are the associated graded groups of a filtration on $H_{2}\left(\pi_{1}(W) ; \mathbb{Z}\right)$. But the latter group is $\mathbb{Z}$ by Proposition 4.3 , so each associated graded group must be cyclic, and since $E_{1,1}^{\infty}$ is cyclic we must have $n=1$.

4.3. The homotopy type of a Stein filling. So far we have shown that if $(W, J)$ is a Stein filling of $\left(Y_{g}, \xi_{g}\right)$, then $W$ has the homology and intersection form of the disk cotangent bundle $D T^{*} \Sigma_{g}$ (Theorem 4.2) and that $\pi_{1}(W) \cong \pi_{1}\left(\Sigma_{g}\right)$ (Theorem4.4), with the circle fiber of $Y_{g}=\partial W$ being nullhomotopic in $W$. In this section we will deduce that $W$ is therefore homotopy equivalent, and thus s-cobordant, to $D T^{*} \Sigma_{g}$ rel boundary.

Proposition 4.9. If $(W, J)$ is a Stein filling of $\left(Y_{g}, \xi_{g}\right)$, then $W$ is aspherical.

Proof. A decomposition of $W$ into handles of index at most 2, with exactly one 0-handle, necessarily has $2 g-1+k$-handles and $k$ 2-handles for some $k \geq 1$ since $\chi(W)=2-2 g$. The corresponding presentation of $\pi_{1}(W) \cong \pi_{1}\left(\Sigma_{g}\right)$ has $2 g-1+k$ generators and $k$ relations and thus deficiency $2 g-1$.

Hillman [Hil97, Proof of Theorem 2] showed that if a presentation $P$ of a group $G$ has deficiency $1+\beta_{1}(G)$, where $\beta_{1}$ denotes the first $L^{2}$-Betti number (see for example [Lüc02]), 
then the 2-complex corresponding to $P$ is aspherical. In the above case we know that $\beta_{1}\left(\pi_{1}\left(\Sigma_{g}\right)\right)=2 g-2$, so the 2-complex corresponding to the given presentation of $\pi_{1}\left(\Sigma_{g}\right)$ is aspherical, and thus $W$ (which retracts onto this complex) is aspherical as well.

We have now shown that $W$ is a $K\left(\pi_{1}\left(\Sigma_{g}\right), 1\right)$, and so it has the homotopy type of $D T^{*} \Sigma_{g}$. Since both are compact 4-manifolds with boundary, we can strengthen this to an assertion about manifolds rel boundary as follows.

Theorem 4.10. If $(W, J)$ is a Stein filling of $\left(Y_{g}, \xi_{g}\right)$, then $W$ is s-cobordant rel boundary to the disk cotangent bundle $D T^{*} \Sigma_{g}$.

Proof. It suffices to find a homotopy equivalence $f: D T^{*} \Sigma_{g} \rightarrow W$ which restricts to a homeomorphism $\partial\left(D T^{*} \Sigma_{g}\right) \stackrel{\sim}{\rightarrow} \partial W$. Since $W$ is compact and aspherical with $\pi_{1}(W)$ a surface group, Khan [Kha12, Corollary 1.23] showed that $W$ is topologically s-rigid, a condition which implies that if such an $f$ exists then $D T^{*} \Sigma_{g}$ is s-cobordant to $W$.

To construct $f: D T^{*} \Sigma_{g} \rightarrow W$, following Stipsicz [Sti02], we first take a standard handlebody decomposition of $D T^{*} \Sigma_{g}$, with a 0-handle, $2 g$ 1-handles, and a single 2-handle, and turn it upside down to build $D T^{*} \Sigma_{g}$ from a thickened $Y_{g}$ by attaching a 2-handle, $2 g$ 3-handles, and a 4-handle. We define $f$ by identifying the boundaries, $\partial\left(D T^{*} \Sigma_{g}\right) \stackrel{\sim}{\rightarrow} \partial W$, in a way which sends a circle fiber to a circle fiber; extending $f$ over the 2-handle of $D T^{*} \Sigma_{g}$, which can be done since the attaching curve is identified with the circle fiber in $Y_{g}=\partial W$ and is thus nullhomotopic in $W$; and then extending $f$ over the 3 - and 4-handles of $D T^{*} \Sigma_{g}$, since the obstructions to doing so lie in $\pi_{2}(W)=0$ and $\pi_{3}(W)=0$.

The map $f$ which we have constructed now induces an isomorphism $f_{*}: \pi_{1}\left(D T^{*} \Sigma_{g}\right) \rightarrow$ $\pi_{1}(W)$, since it induces an isomorphism $\pi_{1}\left(\partial\left(D T^{*} \Sigma_{g}\right)\right) \stackrel{\sim}{\rightarrow} \pi_{1}(\partial W)$ which preserves the subgroup generated by the circle fiber, and both groups are quotients of $\pi_{1}\left(Y_{g}\right)$ by that subgroup. Moreover, $f$ induces an isomorphism on all higher homotopy groups, since these are identically zero, and so $f$ is a homotopy equivalence by Whitehead's theorem.

\section{REFERENCES}

[Ago08] Ian Agol. Criteria for virtual fibering. J. Topol., 1(2):269-284, 2008.

[Bau08] Stefan Bauer. Almost complex 4-manifolds with vanishing first Chern class. J. Differential Geom., 79(1):25-32, 2008.

[Bro82] Kenneth S. Brown. Cohomology of groups, volume 87 of Graduate Texts in Mathematics. Springer-Verlag, New York-Berlin, 1982.

[Etn04] John B. Etnyre. Planar open book decompositions and contact structures. Int. Math. Res. Not., (79):4255-4267, 2004.

[FQ90] Michael H. Freedman and Frank Quinn. Topology of 4-manifolds, volume 39 of Princeton Mathematical Series. Princeton University Press, Princeton, NJ, 1990.

[Fre82] Michael Hartley Freedman. The topology of four-dimensional manifolds. J. Differential Geom., 17(3):357-453, 1982.

[FS95] Ronald Fintushel and Ronald J. Stern. Immersed spheres in 4-manifolds and the immersed Thom conjecture. Turkish J. Math., 19(2):145-157, 1995.

[Gir01] Emmanuel Giroux. Structures de contact sur les variétés fibrées en cercles au-dessus d'une surface. Comment. Math. Helv., 76(2):218-262, 2001.

[Hem72] John Hempel. Residual finiteness of surface groups. Proc. Amer. Math. Soc., 32:323, 1972.

[Hil97] Jonathan A. Hillman. On $L^{2}$-homology and asphericity. Israel J. Math., 99:271-283, 1997.

[Hin00] Richard Hind. Holomorphic filling of RP ${ }^{3}$. Commun. Contemp. Math., 2(3):349-363, 2000.

[Kal13] Amey Kaloti. Stein fillings of planar open books. arXiv:1311.0208, 2013.

[Kha12] Qayum Khan. Homotopy invariance of 4-manifold decompositions: connected sums. Topology Appl., 159(16):3432-3444, 2012. 
[Li06a] Tian-Jun Li. Quaternionic bundles and Betti numbers of symplectic 4-manifolds with Kodaira dimension zero. Int. Math. Res. Not., pages Art. ID 37385, 28, 2006.

[Li06b] Tian-Jun Li. Symplectic 4-manifolds with Kodaira dimension zero. J. Differential Geom., 74(2):321-352, 2006.

[Lis08] Paolo Lisca. On symplectic fillings of lens spaces. Trans. Amer. Math. Soc., 360(2):765-799 (electronic), 2008.

[LMY14] Tian-Jun Li, Cheuk Yu Mak, and Kouichi Yasui. Uniruled caps and Calabi-Yau caps. arXiv:1412.3208, 2014.

[LS91] François Lalonde and Jean-Claude Sikorav. Sous-variétés lagrangiennes et lagrangiennes exactes des fibrés cotangents. Comment. Math. Helv., 66(1):18-33, 1991.

[Lüc02] Wolfgang Lück. $L^{2}$-invariants: theory and applications to geometry and K-theory, volume 44 of Ergebnisse der Mathematik und ihrer Grenzgebiete. 3. Folge. A Series of Modern Surveys in Mathematics [Results in Mathematics and Related Areas. 3rd Series. A Series of Modern Surveys in Mathematics]. Springer-Verlag, Berlin, 2002.

[Lut83] Robert Lutz. Structures de contact et systèmes de Pfaff à pivot. In Third Schnepfenried geometry conference, Vol. 1 (Schnepfenried, 1982), volume 107 of Astérisque, pages 175-187. Soc. Math. France, Paris, 1983.

[McD90] Dusa McDuff. The structure of rational and ruled symplectic 4-manifolds. J. Amer. Math. Soc., 3(3):679-712, 1990.

[McD91] Dusa McDuff. Symplectic manifolds with contact type boundaries. Invent. Math., 103(3):651671, 1991.

[MS97] John W. Morgan and Zoltán Szabó. Homotopy K3 surfaces and mod 2 Seiberg-Witten invariants. Math. Res. Lett., 4(1):17-21, 1997.

[OO05] Hiroshi Ohta and Kaoru Ono. Simple singularities and symplectic fillings. J. Differential Geom., 69(1):1-42, 2005.

[Pol91] L. Polterovich. The surgery of Lagrange submanifolds. Geom. Funct. Anal., 1(2):198-210, 1991.

[PVHM10] Olga Plamenevskaya and Jeremy Van Horn-Morris. Planar open books, monodromy factorizations and symplectic fillings. Geom. Topol., 14(4):2077-2101, 2010.

[Sei08] Paul Seidel. Fukaya categories and Picard-Lefschetz theory. Zurich Lectures in Advanced Mathematics. European Mathematical Society (EMS), Zürich, 2008.

[Sta65] John Stallings. Homology and central series of groups. J. Algebra, 2:170-181, 1965.

[Sta15] Laura Starkston. Symplectic fillings of Seifert fibered spaces. Trans. Amer. Math. Soc., 367(8):5971-6016, 2015.

[Sti02] András I. Stipsicz. Gauge theory and Stein fillings of certain 3-manifolds. Turkish J. Math., 26(1):115-130, 2002.

[Tau95] Clifford Henry Taubes. More constraints on symplectic forms from Seiberg-Witten invariants. Math. Res. Lett., 2(1):9-13, 1995.

[Tau96] Clifford H. Taubes. SW $\Rightarrow$ Gr: from the Seiberg-Witten equations to pseudo-holomorphic curves. J. Amer. Math. Soc., 9(3):845-918, 1996.

[Wen10] Chris Wendl. Strongly fillable contact manifolds and J-holomorphic foliations. Duke Math. J., 151(3):337-384, 2010.

[Wen14] Chris Wendl. A biased survey on symplectic fillings, part 7 (maximal elements and co-fillability). https://symplecticfieldtheorist.wordpress.com/2014/12/29/a-biased-survey-on-symplectic-fillings-part 2014.

E-mail address: ssivek@math.princeton.edu

Department of Mathematics, Princeton University

E-mail address: jvhm@uark.edu

Department of Mathematical Sciences, The University of Arkansas 